\title{
Avaliação de um sistema especialista em diagnóstico de enfermagem relacionados à eliminação urinária*
}

\author{
Evaluation of an expert system on nursing diagnoses related to urinary elimination
}

Evaluación de un sistema especialista en diagnósticos de enfermeria relacionados a la eliminiación urinária

\section{Rosângela Higa', Maria Helena Baena de Moraes Lopes ${ }^{1}$}

'Universidade Estadual de Campinas. Faculdade de Ciências Médicas, Departamento de Enfermagem. Campinas, SP

Submissão: 1 1/07/2007

Aprovação: 15/09/2008

RESUMO

Foi desenvolvido e avaliado um sistema especialista em diagnósticos de enfermagem relacionados à eliminação urinária, segundo a taxionomia da NANDA. Para coleta de dados utilizou-se um roteiro e um checklist com as características definidoras. Os diagnósticos obtidos por consenso entre três especialistas foram considerados padrão-ouro. Foram testados 197 casos. O Sistema mostrou ser adeQuado para a determinação dos diagnósticos 'incontinência urinária (IU) por pressão', 'IU por impulso', 'retenção urinária 'e 'IU total', com sensibilidade e especificidade superiores a 98\%. A pequena casuística não possibilitou avaliar a acurácia em relação à 'eliminação urinária prejudicada', 'IU reflexa' e 'IU funcional'. Esta experiência de desenvolvimento e avaliação poderá ser aplicada na criação de outros sistemas especialistas.

Descritores: incontinência urinária; Sistemas especialistas; Diagnóstico de enfermagem.

\section{ABSTRACT}

An expert system on nursing diagnoses related to urinary elimination, according NANDA's taxonomy, was developed and evaluated. Data were collected using a form and a checklist of defining characteristics. The obtained consensus diagnoses by three specialists were considered gold standard. 197 cases were tested. The system proved to be adeQuate for determining diagnoses such as "stress urinary incontinence', 'urge urinary incontinence', 'urinary retention' and 'total urinary incontinence' with sensitivity and specificity above 98\%. The accuracy evaluation in relation to 'impaired urinary elimination', 'reflex urinary incontinence' and 'functional urinary incontinence' was not possible to be established due to the small size of the sample. The experience in developing and evaluating this program can be applied in creating other expert systems.

Descriptors: Urinary incontinence; Expert systems; Nursing diagnosis.

\section{RESUMEN}

Fue desarrollado y avaluado un sistema especialista en diagnósticos relacionados a la eliminación urinaria, usando la taxonomia de NANDA. Para colectar los datos fue utilizado un programa de entrevista y un checklist conteniendo las características definidoras. Los diagnósticos obtenidos por concordancia de tres especialistas fueron considerados como prototipo-oro. Fueran testados 197 casos. El sistema desarrollado, mostró ser adecuado en la determinación de los diagnósticos 'incontinencia urinaria por presión', 'incontinencia urinaria por impulso', 'retención urinaria' e 'incontinencia urinaria total' con sensibilidad y especificidad superior a $98 \%$. Debido a pequeña casuística, no fue posible avaluar los diagnósticos 'eliminación urinaria perjudicada', 'incontinencia urinaria refleja' e 'incontinencia urinaria funcional'. Esta experiencia de desenvolvimiento e avaluación podrá ser aplicada en la creación de otros sistemas.

Descriptores: Incontinencia urinaria; Sistemas especialistas; Diagnóstico de enfermería.

* Pesquisa financiada pela Fundação de Amparo à Pesquisa do Estado de São Paulo (FAPESP). 


\section{INTRODUÇÃO}

A incontinência é um problema comum de eliminação urinária alterada, principalmente na população feminina. A literatura demonstra Que a prevalência de incontinência urinária varia de 14\% a 57\% entre mulheres com idade entre 20 e 89 anos, com episódios de perda urinária Que variam de esporádicas a diárias ${ }^{(1-4)}$. Entre os homens, a prevalência é bem mais baixa, em geral cerca de $3 \%$ a $11 \%{ }^{(5)}$.

Além dos problemas de ordem física, a IU causa restrições à vida pessoal e profissional das pessoas incontinentes ${ }^{(6)}$ e afeta sua Qualidade de vida ${ }^{(7)}$. Assim, além do tratamento cirúrgico ou medicamentoso, o tratamento comportamental e a orientação Quanto ao manejo adequado do problema devem ser valorizados e implementados tão logo ele seja identificado.

A classificação da North American Diagnosis Association (NANDA), atualmente denominada NANDA Internacional (NANDAI), contempla vários diagnósticos relacionados à eliminação urinária: eliminação urinária prejudicada; incontinência urinária por pressão; incontinência urinária reflexa; incontinência urinária de impulso; risco para incontinência urinária de impulso; incontinência urinária funcional; incontinência urinária total e retenção urinária.

O diagnóstico diferencial envolve a coleta de dados sobre o histórico de saúde focalizando os sintomas do trato urinário inferior e os problemas urinários, o exame clínico e avaliação urodinâmica. Algumas classes de incontinência urinária, por exemplo, são às vezes difíceis de serem estabelecidas e como regra, somente os resultados de exames urodinâmicos permitem uma acurácia diagnóstica adequada $^{(8)}$. O diagnóstico, portanto, pode ser uma tarefa difícil para enfermeiros com formação não especializada na área e um sistema especialista para apoio a decisão pode ser útil nesses casos.

Na prática cotidiana, em especial na assistência primária, muitas vezes não se tem prontamente disponíveis recursos tecnológicos como a avaliação urodinâmica, desta forma a anamnese e o exame físico podem ser de grande valia para identificar o problema urinário. Comumente é solicitada a avaliação urodinâmica Quando uma intervenção cirúrgica é necessária. Quando se realiza o tratamento conservador, nem sempre é feito este exame.

Assim, Quando o enfermeiro generalista identifica um caso de eliminação urinária alterada, ele deve ser capaz de estabelecer o diagnóstico de enfermagem, principalmente, com base nas Queixas clínicas, implementar intervenções Que, pelo menos, minimizem os sintomas, evitando que o cliente maneje de forma inadequada o problema, por ex., restringindo a ingestão de lį́uidos. Caso o enfermeiro não tenha experiência na área, deverá encaminhar a um especialista (enfermeiro, médico ou fisioterapeuta) para Que faça uma avaliação mais acurada.

Há alguns anos foi desenvolvido um sistema de apoio à decisão em enfermagem denominado ALTURIN. EXP(), envolvendo os diagnósticos de enfermagem relacionados à eliminação urinária e utilizando o programa EXPERTMD ${ }^{(10)}$, Que é um sistema "shell", ou seja, é um programa capaz de realizar o mecanismo de inferência, segundo a metodologia de sistemas especialistas de consulta baseados em regra de produção, para QualQuer tipo de domínio de conhecimento. Na criação deste sistema foi utilizada a Taxionomia I Revisada da NANDA, com as modificações propostas no II Simpósio Nacional sobre Diagnóstico de Enfermagem ${ }^{(11)}$.

Passados alguns anos, sentiu-se a necessidade de atualizá-lo. Assim, foi desenvolvido um novo sistema, denominado ALTURIN.SDD ${ }^{(12)}$. O sistema ALTURIN.SDD foi criado utilizando-se um programa "shell" especialmente construído para este projeto, o Programa para Determinação de Diagnósticos (SDD, Versão 1.0), tendo como alvo plataformas Windows 95/98/Me. Os diagnósticos de enfermagem são aQueles presentes na classificação da NANDA 2001-2002. Em um artigo anterior é descrito, com detalhes, como foi desenvolvido o sistema ALTURIN.SDD ${ }^{(12)}$. O presente trabalho teve como objetivo avaliar o desempenho do sistema especialista ALTURIN.SDD.

\section{MÉTODO}

Tratou-se de um estudo prospectivo de validação de teste diagnóstico, cujas etapas são descritas a seguir. A população de estudo foi composta por mulheres com evidências de alteração na eliminação urinária, isto é, com Queixa de incontinência, urgência, noctúria, hesitação, freoüência, disúria ou retenção. Foram excluídos os casos com diagnóstico Risco para incontinência urinária de impulso ${ }^{(13)}$. Os dados foram coletados até se satisfazer o tamanho amostral.

Pela falta de informação a priori, isto é, por não existirem estudos semelhantes na literatura, para o cálculo levou-se em consideração a presença/ausência de cada diagnóstico. Assim, a estimativa por intervalo das freqüências de presença de cada um, considerando-se um nível de significância de 5\% e tolerância de 7\%, resultou em um tamanho amostral de 196 casos, considerando-se uma proporção de presença do diagnóstico de 50\%, ou seja, ąuela de maior variabilidade e Que levaria ao tamanho amostral Que cobriria Qualquer outra estimativa desta proporção. A fim de Que os valores obtidos de sensibilidade e especificidade fossem confiáveis, ao calcular-se o intervalo de confiança o valor de $50 \%$ não poderia estar dentro deste intervalo. $\mathrm{O}$ estudo foi realizado nas unidades de internação de Ginecologia e Oncologia do CAISM e Urologia do Hospital das Clínicas da Universidade Estadual de Campinas (Unicamp).

Os dados foram coletados pelas pesquisadoras através de um instrumento Que continha a identificação da cliente, um roteiro adaptado da proposta de Carpenito ${ }^{(14)}$ Que propõe critérios para o assessment focalizado de casos de eliminação urinária alterada e um checklist Que continha todas as características definidoras dos diagnósticos: eliminação urinária prejudicada; incontinência urinária por pressão; incontinência urinária reflexa; incontinência urinária de impulso; incontinência urinária funcional; incontinência urinária total e retenção urinária. Embora o diagnóstico 'eliminação urinária prejudicada', seja comum a todos os casos, poderiam ocorrer situações em Que não haveria incontinência ou retenção, mas poderiam estar presentes sintomas como disúria, noctúria e outros; somente foi considerada a presença deste diagnóstico nestes casos. Analisandose os cinco primeiros casos (pré-teste), verificou-se não haver necessidade de alterar o instrumento de coleta de dados.

Três casos foram coletados conjuntamente pelas duas pesquisadoras a fim de padronizar a coleta. Antes da análise, os casos foram revistos, fazendo-se, Quando necessário, correções nos registros. Os casos foram discutidos conjuntamente pelas pesquisadoras (uma enfermeira docente e uma assistencial) Que atuam há mais de 15 anos na área de Saúde da Mulher e têm experiência há mais de 10 com a utilização dos diagnósticos de enfermagem da NANDA, com publicações sobre ambos os temas.

Posteriormente, todos os casos foram encaminhados para uma 
especialista em diagnósticos de enfermagem de outra instituição de ensino superior, Que foi consultora deste estudo e é uma das mais renomadas especialistas em diagnóstico de enfermagem de nosso país. Periodicamente foram realizadas reuniões com a consultora e os diagnósticos determinados pelas pesquisadoras e aqueles identificados pela consultora foram confrontados e discutidos. Somente os casos em Que foi possível o consenso foram considerados como padrão-ouro, excluindo-se os demais.

Todos os casos foram testados usando o sistema ALTURIN.SDD. Os diagnósticos definidos pelo Sistema foram comparados com aqueles obtidos por consenso entre as especialistas (padrão-ouro). Foram calculadas a sensibilidade, a especificidade (com os respectivos intervalos de confiança), a acurácia ${ }^{(15)}$.

Para a análise estatística houve assessoria dos estatísticos da Comissão de Pesquisa da Faculdade de Ciências Médicas (FCM) Unicamp e da Comissão de Pesquisa do CAISM - Unicamp. Foram utilizados os programas computacionais SAS System for Windows
(Statistical Analysis System), versão 8.1 e Microsoft Excel 2000 da Microsoft Corporation.

Quanto aos aspectos éticos, foi solicitado o consentimento informado por escrito junto aos clientes e mantido sigilo Quanto à identificação dos mesmos durante o transcorrer do estudo. O projeto foi aprovado pela Comissão de Pesquisa do CAISM e Comitê de Ética em Pesquisa da FCM - Unicamp (Parecer no 19/99).

\section{RESULTADOS}

No período de fevereiro de 2000 a junho de 2002 foram entrevistados 202 clientes. Foram eliminados cinco casos por não ser haver consenso. A amostra foi constituída, portanto, por 197 casos, sendo três homens e 194 mulheres, com idade média de 50,83 anos, mínima de 25 e máxima de 85 anos.

A Tabela I apresenta os diagnósticos de enfermagem identificados pelas especialistas por consenso (padrão-ouro).

Tabela 1. Categorias diagnósticas Que foram identificadas por enfermeiras especialistas, segundo a Taxionomia II da NANDA entre clientes internados em clínicas de ginecologia, oncologia ginecológica e urologia. Campinas, SP. 2000-2002.

\begin{tabular}{llc}
\hline Diagnósticos de Enfermagem & n & \% \\
\hline Incontinência urinária por pressão + Incontinência urinária de impulso (IUP+IUI) & 97 & 49,3 \\
Incontinência urinária por pressão (IUP) & 54 & 27,4 \\
Incontinência urinária de impulso (IUI) & 17 & 8,6 \\
Retenção urinária (RU) & 14 & 7,1 \\
Incontinência urinária total (IUT) & 12 & 6,1 \\
Eliminação urinária prejudicada (EUP) & 2 & 1,0 \\
Incontinência urinária reflexa (IUR) & 1 & 0,5 \\
\hline Total & 197 & $\mathbf{1 0 0 , 0}$ \\
\hline
\end{tabular}

Tabela 2. Comparação entre as categorias diagnósticas determinadas pelo Sistema ALTURIN.SDD e pelas enfermeiras especialistas, de acordo com o diagnóstico de enfermagem. Campinas, SP, 20002002.

\begin{tabular}{lcccccccc}
\hline & \multicolumn{7}{c}{ Enfermeiras Especialistas } \\
\cline { 2 - 9 } Sistema ALTURIN.SDD & IUP+IUI & IUP & IUI & RU & IUT & EUP & IUR & Total \\
\hline IUP+IUI & 96 & I & 0 & 0 & 0 & 0 & 0 & 97 \\
IUP & 1 & 53 & 0 & 0 & 0 & 0 & 0 & 54 \\
IUI & 0 & 0 & 17 & 0 & 0 & 0 & 0 & 17 \\
RU & 0 & 0 & 0 & 14 & 0 & 0 & 0 & 14 \\
IUT & 0 & 0 & 0 & 0 & 12 & 0 & 0 & 12 \\
EUP & 0 & 0 & 0 & 0 & 0 & 2 & 0 & 2 \\
IUR & 0 & 0 & 0 & 0 & 0 & 0 & 1 & 1 \\
\hline Total & 97 & 54 & 16 & 13 & 12 & 2 & 1 & 197 \\
\hline
\end{tabular}

Tabela 3. Sensibilidade, especificidade e acurácia do Sistema ALTURIN.SDD, de acordo com a categoria diagnóstica. Campinas, SP. 2000-2002.

\begin{tabular}{|c|c|c|c|}
\hline $\begin{array}{c}\text { DIAGNÓSTICOS DE } \\
\text { ENFERMAGEM }\end{array}$ & $\begin{array}{c}\text { SENSIBILIDADE } \\
\%\end{array}$ & $\begin{array}{c}\text { ESPECIFICIDADE } \\
\%\end{array}$ & $\begin{array}{c}\text { ACURÁCIA } \\
\%\end{array}$ \\
\hline IUP+IUI & 98,97 (IC: 94-100) & 99,00 (IC: 94-100) & 98,98 \\
\hline IUP & 98,15 (IC: 89-100) & 99,30 (IC: 96-100) & 98,98 \\
\hline IUI & 100,00 (IC: 77-99) & $100,00$ (IC: $97-100)$ & 100,00 \\
\hline RU & 100,00 (IC: 73-99) & $100,00$ (IC: $97-100)$ & 100,00 \\
\hline IUT & 100,00 (IC: 70-99) & $100,00$ (IC: $97-100)$ & 100,00 \\
\hline EUP & 100,00 (IC: 20-95) & 100,00 (IC: 98-100) & 100,00 \\
\hline
\end{tabular}


Na Tabela 2 é apresentada a comparação entre os diagnósticos determinados pelo Sistema ALTURIN.SDD e pelas especialistas e na Tabela 3, a sensibilidade, especificidade e acurácia do Sistema. O índice de acerto foi de $98,97 \%$.

\section{DISCUSSÃO}

Verificou-se que o sistema especialista ALTURIN.SDD é adequado de alguns dados, porque nem sempre é possível obter todas as informações e/ou responder com absoluta certeza 'sim'ou 'não' à presença de um sinal ou sintoma (característica definidora).

Pelo fato da avaliação ter sido baseada na Queixa clínica, as enfermeiras especialistas e o Sistema ALTURIN.SDD identificaram uma proporção elevada de casos em Que havia concomitância dos diagnósticos 'incontinência por pressão' e 'incontinência de impulso' (49,3\%), considerados na literatura médica como 'incontinência mista'. Estes achados estão condizentes com os de outros estudos. Na Noruega, um estudo de coorte prospectiva envolvendo 3198 mulheres (2845 responderam ao Questionário), a sintomatologia baseada nas respostas apresentadas pelas pacientes evidenciou índices de 64\%, 24\% e 9\%, para incontinência mista, por esforço e urge-incontinência, respectivamente ${ }^{(19)}$. Em nosso meio, um estudo retrospectivo baseado no atendimento de 114 pacientes, observou Que a Queixa de perda urinária isolada foi referida por 41 pacientes (36,0\%), a urge-incontinência isolada por $13(11,4 \%)$ e os sintomas mistos por $60(52,6 \%)^{(20)}$.
O sistema ALTURIN. SDD apresentou sensibilidade e especificidade elevadas e indicou o diagnóstico correto em Quase 99\% dos casos. Seu desempenho foi comparável ao de sistemas especialistas mais complexos como o sistema denominado Galactica, Que utilizou algoritmos genéticos para descobrir regras para um sistema especialista a partir de bases de dados. Ele foi capaz de identificar regras Que permitiam o diagnóstico correto de incontinência urinária feminina de esforço, mista e de urgência em 89, 86 e $87 \%$ dos casos, respectivamente ${ }^{(18)}$.

Apesar dos resultados iniciais promissores, como a maioria dos casos não tinha resultado de exame urodinâmico recente, o desempenho do sistema ALTURIN.SDD poderia ser diferente na presença desses dados. É importante, portanto, continuar o aprimoramento deste sistema.

\section{CONCLUSÃO E CONSIDERAÇÕES FINAIS}

O sistema desenvolvido apresentou bom desempenho, mas deve ser aperfeiçoado, antes do seu uso mais amplo na prática clínica.

Acredita-se que o sistema especialista desenvolvido poderá ser útil em aplicações no ensino e na prática de enfermagem na área de assistência à saúde da mulher. Para utilização em outras clientelas, faz-se necessário desenvolver estudos semelhantes a este, a fim de validar seu uso para identificação de outros diagnósticos relacionados à eliminação urinária, Que pela baixa freeüência na população estudada, não puderam ser identificados e avaliados.

\section{REFERÊNCIAS}

I. Bjornsdóttir LT, Geirsson RT, Jónsson PV. Urinary incontinence and urinary tract infections in octogenarian women. Acta Obstet Gynecol Scand 1998; 77: 105-09.

2. Guarisi T, Pinto-Neto AM, Pedro AO, Costa-Paiva LH, Faundes A. Sintomas urinários e genitais em mulheres climatéricas. I Bras Ginecol 1998; 108(4): 125-30.

3. Simeonova Z, Milsom I, Kullendorff AM, Molander U, Bengtsson C. The prevalence of urinary incontinence and its influence on the Quality of life in women from an urban Swedish population. Acta Obstet Gynecol Scand 1999; 78: 546-51.

4. Sampselle CM, Harlow SD, Skurnick IS, Brubaker L, Bondarenko I. Urinary incontinence predictors and life impact in ethnically diverse perimenopausal women. Obst Gynecol 2002; 100(6): 1230-38.

5. Nitti VW. The prevalence of urinary incontinence. Rev Urol 200 I;3(suppl I): S2-S6

6. Fitzgerald ST, Palmer MH, Berry SI, Hart K. Urinary Incontinence. Impact on working women. AAOHN I 2000; 48(3): 1 12-8.

7. Kelleher CI, Cardozo LD, Khullar V, Salvatore S. A new Questionnaire to assess the Quality of life of urinary incontinent women British I Obst Gynaecol 1997; 104: 1374-9.

8. Lurikkala J, Juhola M, Lammi S, Penttinen J, Aukee P Analysis of the imputed female urinary incontinence data for the evaluation of expert system parameters. Computers Biol Med 200 I ; 3 I (4): 239-57.

9. Lopes MHBM, Teixeira JM, Freitas MRR. Apoio à decisão em enfermagem nos casos de eliminação urinária alterada: Sistema Alturin Exp. Rev Bras Enferm 1997; 50(2): 163-8.
10. Sabbatini RME. EXPERTMD: manual de uso. $4^{\mathrm{a}}$ ed. Campinas: Núcleo de Informática Biomédica/Unicamp; 1992.

11. Nóbrega MML, Garcia TR, organizadoras. Uniformização da linguagem dos diagnósticos de enfermagem da NANDA: sistematização das propostas do II SNDE. João Pessoa: A União, CNRDE/GIDE - PB; 1994. p. 32-38.

12. Lopes MHBM, Higa R. Desenvolvimento de um sistema especialista para identificação de diagnósticos de enfermagem relacionados com a eliminação urinária. Rev Bras Enferm 2005; 58(1): 27-32.

13. North American Nursing Diagnosis Association. Diagnósticos de enfermagem da NANDA: definições e classificação - 20012002. Porto Alegre: Artmed; 2002.

14. Carpenito LJ. Diagnóstico de enfermagem: aplicação à prática clínica. 6a ed. Porto Alegre: Artmed; 1996.

15. Fletcher R, Fletcher S, Wagner EH. Epidemiologia Clínica. 2ª ed. Porto Alegre: Artes Médicas; 1991.

16. Lurikkala J, Juhola M, Lammi S, Penttinen J, Aukee P Analysis of the imputed female urinary incontinence data for the evaluation of expert system parameters. Comput Biol Med 200 I ; 3 (4): 289-57.

17. Riss PA, Koelbl H. Development of an expert system for preoperative assessment of female urinary incontinence. Int I Biomed Comput 1988; 22(3-4): 21 17-23.

18. Laurikkala J, Juhola M. A genetic-based machine learning system to discover the diagnostic rules for female urinary incontinence. Comput Methods Programs Biomed 1998; 55(3):2 17-28. 
19. Indrekvam S, Fosse OAK, Hunskaar SA. Norwegian national cohort of 3198 women treated with home-managed electrical stimulation for urinary incontinence. Scand I Urol Nephrol 2000: 35: 26-31.
20. Feldner Ir PC, Bezerra LRPS, Girão MJBC, Castro RA, Sartori, MGF, Baracat EC, et al. Valor da Queixa clínica e exame físico no diagnóstico da incontinência urinária. Rev Bras Ginecol Obstet 2002; 24(2): 87-91. 